\title{
Pulse Position modulation based Energy Detection for Smart Grid Communication
}

\author{
Haroon Rasheed \\ Department of Electrical Engineering \\ Bahria University \\ Karachi Pakistan \\ haroonrasheed.bukc@bahria.edu.pk
}

\author{
Farah Haroon \\ Institute of Industrial Electronics \\ Pakistan Council for Sci and Ind Research \\ Karachi Pakistan \\ farah@iiee.edu.pk
}

\author{
Nandana Rajatheva \\ Center of Wireless Communication \\ Oulu University 90570 \\ Oulu, Finland \\ rrajathe@ee.oulu.fi
}

\begin{abstract}
Smart Grid Network (SGN) concept is developed for dynamically and automatically respond to changes in power grid conditions, which will require sensors to provide detection of signal for real-time information and status. The most distinguishing attribute of Pulse Position Modulation (PPM) in SG Communications is its non coherent detection. It avoids complex channel estimation which becomes a very complicated process due to very large bandwidth and fine time resolution with large number of multipath components. In this paper, we analyze PPM based energy detection for sensing in smart grid networks where intelligent devices for power monitoring, smart sensors and smart meters for Home Area Network (HAN) or Neighborhood Area Network (NAN) can start transmission at any time. In contrast to well known case of Additive White Gaussian Noise (AWGN) channel, we further extended our investigation to $K_{G}$ composite model and the effect of fading and shadowing severity is discussed extensively. Reduction in either the fading index $m$ or shadowing parameter $m_{0}$ influences the channel characteristics and declines the error performance. Hence, the presented results furnish guidelines for energy detection based sensing of PPM modulated signal for SGN various communication models.

Index Terms-PPM; Energy Detection; Smart Grid Network; HAN; NAN; WAN.
\end{abstract}

\section{INTRODUCTION}

Smart Grid Network (SGN) is the next-generation power network expected to significantly increase renewable energy capacity, improve reliability, produce low latency times, support multi connectivity over micro grids, and increase the control efficiency of transmission and distribution network elements. Promising to exhibit the characteristics and attributes of present intelligent communication networks, smart grid and micro grid networks often categorize as Energy Internet as shown in Fig 1.

In general, to ensure transparent connectivity from consumers home to generation plant, three domains of communications are being proposed for smart grid networks [1]. Based on coverage and operating perspective smart grid consists of, Home Area Network (HAN), Neighborhood Area Network (NAN) or Field Area Network (FAN), and Wide Area Network (WAN) as depicted in Fig. 2. Coupled with power consumption control tool, energy storage devices, renewable energy units, energy displays panels, and smart metering facilities in order to ensure transparent connectivity to all in-house components. HAN is aimed for residential unit communications that op-

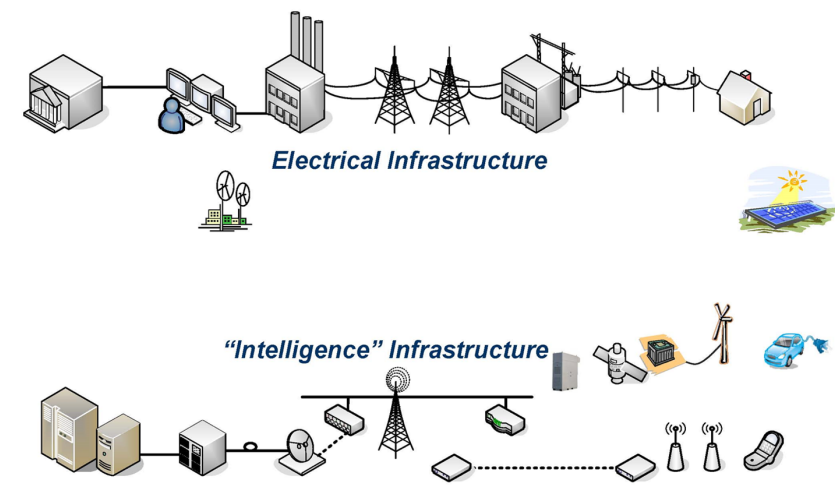

Fig. 1. Power Network comparison to Communication Network.

erates with both RF and IP-based networks, including Blue tooth, WiFi, WiMAX, and GPRS/cellular, or Power Line Communication (PLC), either individually or in combination.

This permits an electric utility to make further information flow between NAN or FAN (based on Intelligent Electronics Devices(IEDs)) e.g., real time monitoring and control, demand response, user information, prepaid and postpaid services between various actors in the energy generation, supply and utilization system. Finally, Wide Area Network (WAN) covers long-haul distances from NAN/FAN to a control center and provide support for real-time monitoring, control and protection applications, communication links for smart grid backbones to improve power system planning [2]. This implies that to detect a fault, or to update a device status, a sensor must frequently sample the signal and compare its characteristics to pre-set trigger levels. Sensors are employed to determine an extensive variety of physical parameters in power generation and plant operations, power transmission lines, distribution substations, power distribution lines, energy storage, and customers premises [3] [4].

An energy detector can collect the multipath energy very easily by means of a simple integrator without any channel estimation. The timing accuracy is also acceptable and the BER is not largely affected due to synchronization errors. Hence, they render low complexity and low power consumption solutions for SGN devices detection and are suitable 


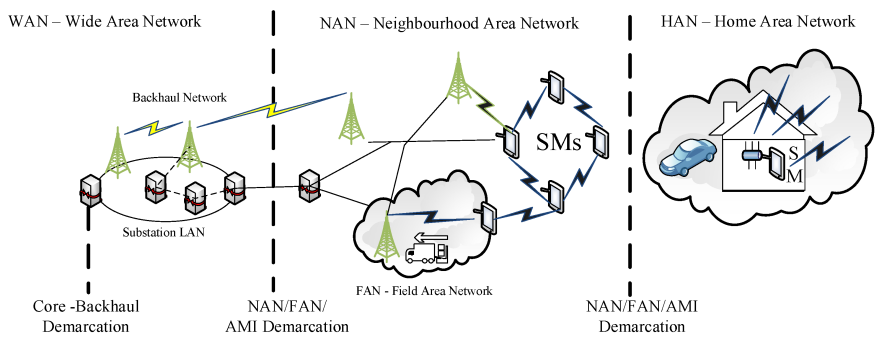

Fig. 2. Smart Grid Communication Architecture [5]

for peer-to-peer communications. Energy detection with pulse position modulation (PPM) is presented for sensing analysis in Smart Grid networks. The most distinguishing attribute of PPM in communication systems is its non coherent detection [6]. It avoids complex channel estimation which becomes a very complicated process due to very large bandwidth and fine time resolution with large number of multipath components. Implementation simplicity of PPM through energy detection and controlling delays for interference cancellation provides better spectral manipulation. IEEE 802.15.4a standard has suggested that the hybrid BPSK-PPM modulation can be used for noncoherent systems. Multi hops relaying protocol to provide high bit rate using PPM modulation is discussed in [7]. Whereas, noncoherent pulse-position modulation (PPM) is investigated to achieve ultra-low power wireless realizations in WBAN [8]. The results reported in [9] and references show that compared with the BPSK modulation, a hybrid BPSKPPM modulation improves both BER performance and transmission rate in UWB communication. In [10], the complexity of coherent receivers is addressed by noncoherent energy detector associated with M-ary PPM using space time coding for MIMO systems.

Compared to coherent detection the efforts with respect to the receiver synchronization are considerably reduced. Hence, it avoids the problem of nonlinearity of the analog modulation and improves the SNR over analog modulation. On the other hand, it is not purely digital, therefore no pulse-code modulation (PCM) coding is required. Due to fixed symbol interval, signals are much easier to multiplex and demultiplex in the time domain. Therefore, binary PPM is the promising essential modulation technique for future low power networks [11].

Non coherent ED based reception of PPM signals is addressed in [12] for UWB applications. It is akin to a generalized maximum likelihood (GML) detector where the received signal energy in $\mathrm{M}$ possible pulse positions during an observation interval is compared and the position with the maximum energy is estimated and declared. Despite this, these suboptimal detectors offer the wideband Gaussian channel capacity for sufficiently large bandwidth.

In this paper we analyze PPM based energy detection for sensing in smart grid networks where intelligent devices for power monitoring, smart sensors and smart meters for Home
Area Network (HAN)or Neighborhood Area Network (NAN) can start transmission at any time. It is an optimal scheme, whenever the arrival time of a signal is unknown.

\section{PPM BASEd ENERGy Detection}

When time parameter of a signal pulse train is modulated with constant amplitude corresponding process is designated as pulse-position modulation. For PPM, information is expressed in terms of the time interval where the pulse is transmitted. A block diagram of a PPM based energy detection scheme is illustrated in Fig. 3. Taking binary PPM as a simplest case, the duration of the bit interval is divided into two equal time slots $\frac{T_{0}}{2}$, where $T_{0}$ is the bit signaling interval for binary PPM selected from the data rate [13]. Fig. 4 depicts an instance of multi PPM. A signaling interval of $T_{0}$ is separated into equally spaced $D$ sub intervals, each of length $T$ seconds. Conventional PPM involves a pulse that conveys information based on the position when it is not zero. Thus, every $D$ of the pulse positions represents to one message [14]. Once the required message to be transmitted is selected, the pulse position encoder takes decision by the pulse position that exhibits more energy than other positions.

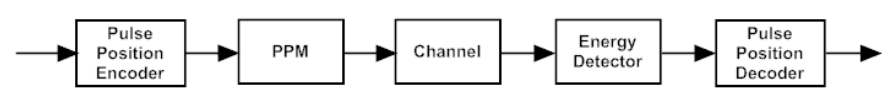

Fig. 3. PPM based non-coherent detection.

\section{System ModeL}

In energy detection for a specified $T$, the transmitted signal $x(t)$ can be written as sum of the square of $2 u$ Gaussian random variables at output signal $Y$ as given in [15]

$$
\begin{aligned}
Y & =\frac{2}{N_{0}} \int_{0}^{T} x^{2}(t) d t \\
& =\sum_{i=1}^{2 u}\left(\frac{h_{i} x_{i}+n_{i}}{\sqrt{N_{0} W}}\right)^{2}: H_{1} \\
& =\sum_{i=1}^{2 u}\left(\frac{n_{i}}{\sqrt{N_{0} W}}\right)^{2}: H_{0}
\end{aligned}
$$

where $N_{0}$ is the Gaussian noise spectral density, $u=T W$ is the product of time and bandwidth, $H_{0}$ and $H_{1}$ are representing transmitted signal absence and presence respectively.

As described in [15], when signal is present decision statistic of $Y$ represents $\chi_{2 u}^{2}$ a central chi square distribution with a with a non centrality parameter $2 \gamma$. Hence, the PDF distribution of test statistic $Y_{i}$, where $i=1,2, \ldots D$ i.i.d random variables when no signal is transmitted, is given as

$$
f_{H_{0}}(y)=\frac{1}{2^{u} \Gamma(u)} y^{u-1} e^{-\frac{y}{2}}
$$

where $\Gamma($.$) is the gamma function.$ 
Similarly, PDF of $Y_{s}$, where $s=1,2, \ldots D$ are i.i.d random variables when signal is present, can be obtained as

$$
f_{H_{1}}(y)=\frac{1}{2}\left(\frac{y}{2 \gamma}\right)^{\frac{u-1}{2}} e^{-\frac{2 \gamma+y}{2}} I_{u-1}(\sqrt{2 \gamma y})
$$

here $I_{v}($.$) is the modified Bessel function of v^{t} h$ order. The received SNR $\gamma$ is defined as $\frac{E_{s}}{N_{0}}$.

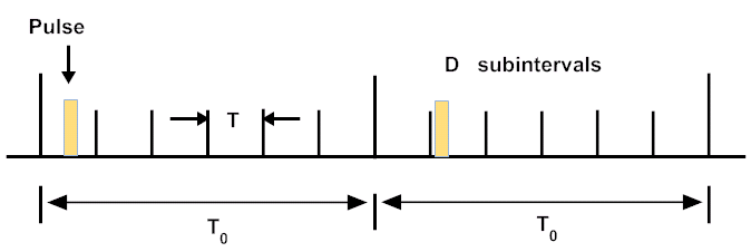

Fig. 4. Signal pulse timing schematic.

\section{A. Error Probability of Energy Detection}

The probability of error is simple when the received signal is less than zero for any $i \neq s$. For $u \geq 1$, the probability of error is given by

$$
P(e)=1-\int_{0}^{\infty}\left[\int_{0}^{\lambda} f_{H_{0}}(y) d y\right]^{D-1} f_{H_{1}}(\gamma) d \gamma
$$

where $f_{H_{1}}(\gamma)$ is the PDF of $Y_{s}$ when there is a threshold $\lambda$ is taken for comparison the received signal.

When device signal is present, Bessel function in $f_{H_{1}}(y)$ can be expanded term by term and expressed as series form of Marcum- $Q$ function as shown in [16]

$$
\begin{array}{r}
\int_{0}^{\infty} f_{H_{1}}(y) d y=\sum_{n=0}^{\infty} \frac{\gamma^{n} e^{-\gamma}}{n !} \\
\sum_{j=0}^{n+u-1} \frac{e^{\frac{-\lambda}{2}}}{j !}\left(\frac{\lambda}{2}\right)^{j}
\end{array}
$$

after simplification the exact probability of error for PPM based energy detection is given by

$$
\begin{array}{r}
P(e)=1-\frac{1}{2} \int_{0}^{\infty}\left[1-e^{\left(\frac{-\lambda}{2}\right)} \sum_{j=0}^{u-1} \frac{1}{j !}\right. \\
\left(\frac{\lambda}{2}\right)^{j D-1}\left(\frac{\lambda}{\gamma}\right)^{(u-1) / 2} e^{-(\lambda+\gamma) / 2} I_{u-1}(\sqrt{\gamma \lambda}) d \gamma
\end{array}
$$

For time bandwidth product $u=1$, the closed-form solution for error probability can be approximated as [16]

$P(e)=1-\left(\frac{1}{D}\right) \sum_{j=1}^{D} \frac{D !}{j !(D-j) !}(-1)^{j+1} \exp \left[\frac{-\gamma(j-1)}{2 j}\right]$

From [17] the closed-form SNR PDF of $K_{G}$ fading is given as

$$
\begin{gathered}
f_{\gamma}(\gamma)=\frac{2}{\Gamma(m) \Gamma\left(m_{0}\right)}\left(\frac{c_{0}}{2}\right)^{m_{0}+m} \\
\gamma^{\left(\frac{m_{0}+m}{2}\right)-1} K_{m_{0}-m}\left(c_{0} \sqrt{\gamma}\right) \quad \gamma>0,
\end{gathered}
$$

where $\Gamma($.$) is the gamma function, scaling parameter c_{0}=$ $2 \sqrt{\frac{m m_{0}}{\bar{\gamma}_{0}}}$ is related to SNR $\gamma, m_{0}$ is the order of Gamma PDF which reflects the shadowing severity, $m$ represents the Nakagami fading parameter, and $K_{m_{0}-m}($.$) is the modified$ Bessel function of order $\left(m_{0}-m\right)$.

From this point, for simplicity we consider $S(e)=1-P(e)$. To compute error probability over $K_{G}$ fading for $u=1$, by averaging (7) with respect to (8) as given by

$$
P_{K_{G}}(e)=\int_{0}^{\infty} S(e) \times f_{\gamma}(\gamma) d \gamma
$$

when substitution (10) expresses the probability of error over $K_{G}$ fading as

$$
\begin{array}{r}
P_{K_{G}}(e)=\frac{2}{\Gamma(m) \Gamma\left(m_{0}\right) D}\left(\frac{c_{0}}{2}\right)^{m_{0}+m} \\
\int_{0}^{\infty}\left[\sum_{j=1}^{D} \frac{D !}{j !(D-j) !}(-1)^{j+1} \exp \left(\frac{-\gamma(j-1)}{2 j}\right)\right] \\
\times \gamma^{\left(\frac{m_{0}+m}{2}\right)-1} K_{m_{0}-m}\left(c_{0} \sqrt{\gamma}\right) d \gamma
\end{array}
$$

It should be noted that (10) is valid only for time bandwidth product $u=1$ state, whereas (6) can be used for computing error probability $u>1$ condition.

\section{Results And Discussion}

In this section, we investigate the performance of PPM based energy detection based on numerical results. For better understanding plots of probability of error estimation versus SNR, time bandwidth product, threshold levels and time interval are presented. In comparison with AWGN, $K_{G}$ fading channel is also employed to highlight shadow-fading realization. The error equations can be computed easily by software package Mathematica 7.0 .

For several fixed values of $\operatorname{SNR} \gamma$, error probability $P(e)$ is evaluated as a function of sub intervals $D$ as shown in Fig. 5. It is evident from plot curves, that large $D$ values require high $\mathrm{SNR}$ for less $P(e)$. The plot is obtained for $u=1$ by using (7) over AWGN. Hence, for better detection under low SNR regions $D$ should be kept minimum.

$P(e)$ in contrast to time bandwidth product $u$ is illustrated in Fig. 6 for several fixed values of SNR $\gamma$. For $u>1$ measure, (6) is used while setting $D=5$. It can be noted from plot curves that $u>5$, there is no variation in $P(e)$ and the curve is almost constant over $\gamma_{d B}=0-10$. This corresponds that for large values of $u$ detector performance deteriorates.

Fig. 7 depicts $P(e)$ variations over threshold values $\lambda=10,20,30$ with two sub intervals $D=2,5$. It can be evident as expected, higher threshold value with minimum $D$ exhibits less errors. This can also be seen that large values of $u$ performance can be make better with large lambda with $D<5$. 
Over show-fading environment $P(e)$ variations as a function of SNR $\gamma$ are demonstrated in Fig. 8. We assume shadowing factor $m_{0}=18.41$ for moderate shadowing and fading severity $m=1$ for several multipath where time bandwidth product $u=1$ . By using (10) PPM based energy detection performance over $K_{G}$ fading is determined. AWGN curves for similar sub intervals $D$ are also provided for comparison. PPM based energy detector error probability increases under more severe shadow-fading conditions higher $D$ values. Combining techniques without channel estimation are needed for acquiring energy arrive from several multipath.

In Fig. 9, we compare different $m_{0}$ and $m$ values taking $u=1$ for $P(e)$ performance analysis. Over $m>1$, detector error performance improves while assuming $D=5$ and $u=1$. Furthermore, shadowing factor $m_{0}$ decreases, error performance degradation increases.

From numerical results and plots, it is observed that in PPM based energy detection $P(e)$ increases monotonically for increased $D, u$ values. It is desirable to employ $D, u$ lower as possible, in order to prevent large SNR penalties for detector operation using PPM modulation. Also, combining techniques can be used for performance improvement.

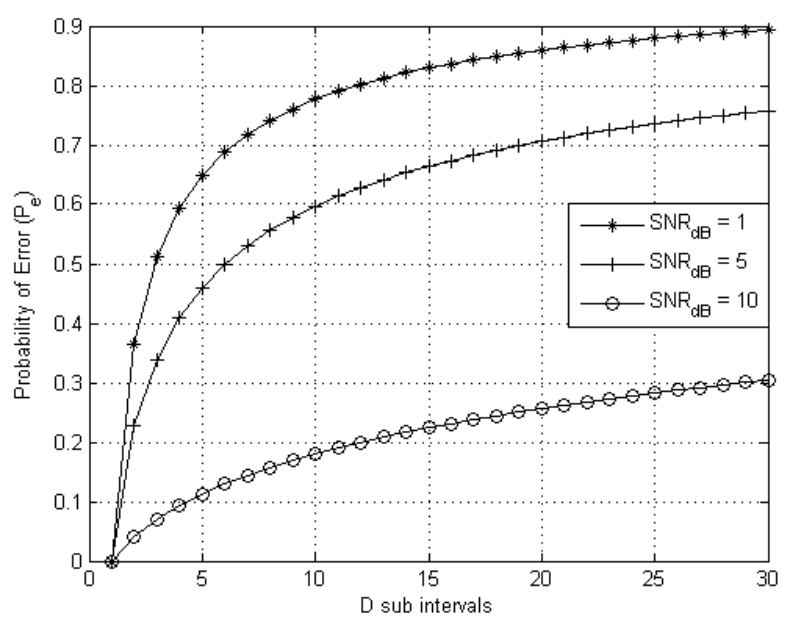

Fig. 5. Probability of error $P(e)$ as a function of sub intervals $D$ for different SNR values where time bandwidth product $u=1$.

\section{CONClusion}

In addition to cognitive radio, this paper brings in the significance of energy detection in Smart Grid Communication systems. With a recent breakthrough of SGN communication, PPM modulation is now gaining popularity at an unprecedented rate. It is one of the promising and attractive candidates for the modulation of sensing signals, when an intricate and difficult channel estimation needs to be averted. Moreover, energy detectors are less susceptible to synchronization and acquisition errors which are the major problems of coherent reception. Analysis process is based on threshold selection, time bandwidth product and sub intervals size by focusing

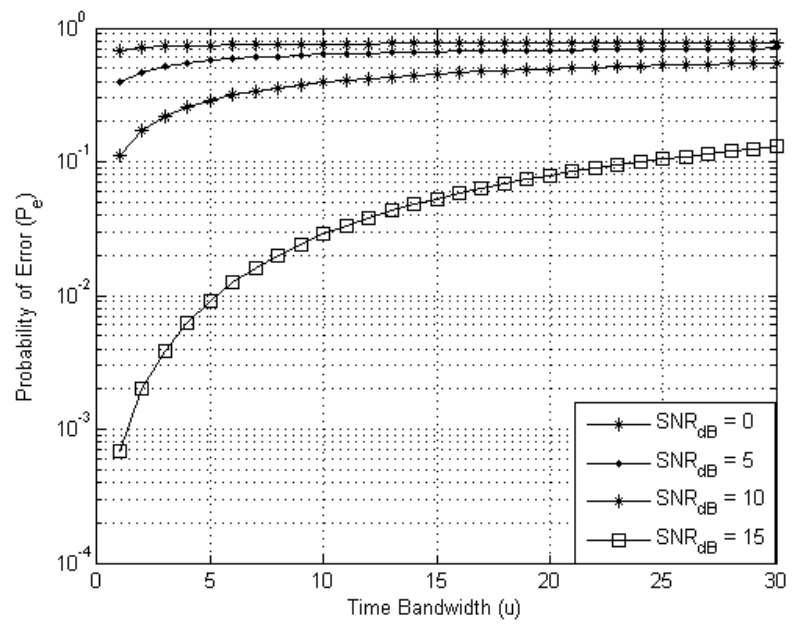

Fig. 6. $P(e)$ as a function of $u$ for several SNR values taking sub intervals duration $D=5$.

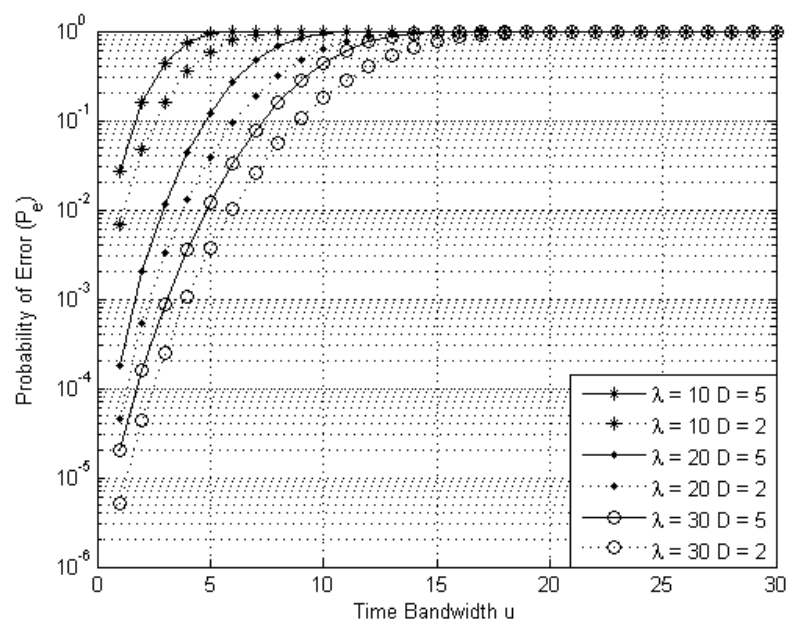

Fig. 7. $P(e)$ as a function of $u$ for various threshold $\lambda$ and sub intervals $D$ values when $\mathrm{SNR} \bar{\gamma}=10 \mathrm{~dB}$.

on probability of error. The obtained numerical plots indicate that an increase in the number of sub intervals or the time bandwidth product always leads to degradation of the error performance. However, SNR improvement in either case augments the probability of error. The effect of sensing threshold is evinced as previously, i.e. a larger $\lambda$ diminishes the probability of error. Hence, non coherent energy detection is a proficient choice for the detection of device signal in SGN systems irrespective of the employed bandwidth and is capable to collect energy from all the multipath components.

\section{ACKNOWLEDGMENT}

The authors would like to thank Higher Education Commission of Pakistan and Bahria University for providing grant and support for this research. 


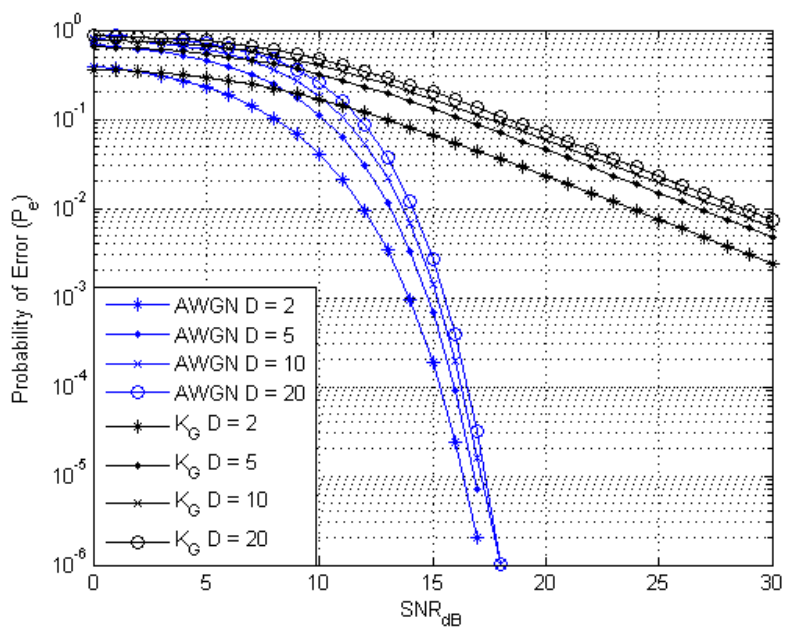

Fig. 8. Probability of error over $K_{G}$ fading $P_{K_{G}}(e)$ versus SNR $\gamma$ for certain sub intervals $D$ while keeping $u=1, m_{0}=18.41$ and $m=1$.

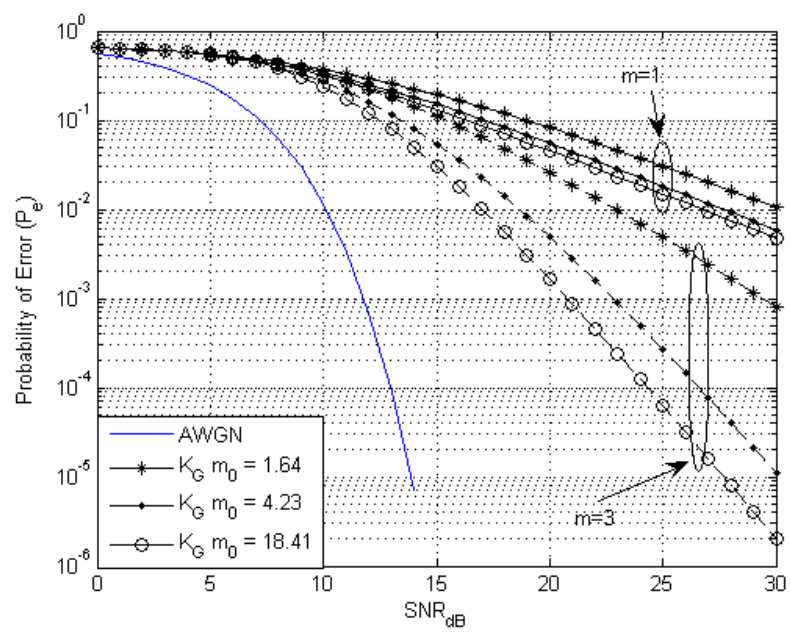

Fig. 9. $P_{K_{G}}(e)$ versus SNR $\gamma$ for various shadowing factor $m_{0}$ values taking fading severity index $m=1,2$ and $D=5$.

\section{REFERENCES}

[1] H. Gabbar, Smart Energy Grid Engineering. Elsevier Science, 2016. [Online]. Available: https://books.google.com.pk/books $\mathrm{id}=\mathrm{VEq0CwAAQBAJ}$

[2] J. Ekanayake, N. Jenkins, K. Liyanage, J. Wu, and A. Yokoyama, Smart Grid: Technology and Applications. Wiley, 2012. [Online]. Available: https://books.google.com.pk/books id=dihQlKa_9YIC

[3] M. Kuzlu, M. Pipattanasomporn, and S. Rahman, "Communication network requirements for major smart grid applications in han, nan and wan," Elsevier Journal of Computer and Telecommunications Networking, , vol. 67, p. 7488, 072014.

[4] E. Y. Song, G. J. FitzPatrick, and K. B. Lee, "Smart sensors and standard-based interoperability in smart grids," IEEE Sensors Journal, vol. 17, no. 23, pp. 7723-7730, Dec 2017.

[5] B. Alohali, K. Kifayat, Q. Shi, and W. Hurst, "Group authentication scheme for neighbourhood area networks (nans) in smart grids," Journal of Sensor and Actuator Networks, vol. 5, no. 2, 2016.

[6] H. Celebi and H. Arslan, "Cross-modulation interference and mitigation technique for ultrawideband ppm signaling," Vehicular Technology, IEEE Transactions on, vol. 57, no. 2, pp. 847 -858, march 2008.
[7] H. Saidi, K. Tourki, and N. Hamdi, "Performance analysis of ppm modulation in multihop fso system over gamma gamma channel," in 2016 International Symposium on Signal, Image, Video and Communications (ISIVC), Nov 2016, pp. 207-212.

[8] P. Zhang, F. M. J. Willems, and L. Huang, "Ppm-based relay communication schemes for wireless body area networks," in 2012 International Symposium on Communications and Information Technologies (ISCIT), Oct 2012, pp. 155-160.

[9] X. Zhang, Z. Liang, H. Liu, D. Meng, and J. Wang, "Weighted autocorrelation detection for trpc-uwb communication systems using hybrid bpsk-ppm modulation," in 2016 5th International Conference on Computer Science and Network Technology (ICCSNT), Dec 2016, pp. 493-496.

[10] C. Abou-Rjeily, "Permutation-based noncoherent space-time codes with analog energy detection for ir-uwb communications with ppm," IEEE Transactions on Wireless Communications, vol. 15, no. 8, pp. 55415554, Aug 2016.

[11] J. Choi and W. Stark, "Performance of ultra-wideband communications with suboptimal receivers in multipath channels," Selected Areas in Communications, IEEE Journal on, vol. 20, no. 9, pp. 1754 - 1766, dec 2002.

[12] C. Carbonelli and U. Mengali, "M-ppm noncoherent receivers for uwb applications," Wireless Communications, IEEE Transactions on, vol. 5, no. 8, pp. $2285-2294$, aug. 2006.

[13] J. Proakis G and M. Salehi, Digital communications. McGraw Hill Higher Education New York:, 2008.

[14] D. Tse and P. Viswanath, Fundamentals of wireless communication. New York, NY, USA: Cambridge University Press, 2005.

[15] H. Urkowitz, "Energy detection of unknown deterministic signals," Proc. IEEE, vol. 55, no. 4, pp. 523-531, 1967.

[16] G. Dillard, "Pulse-position modulation based on energy detection," Aerospace and Electronic Systems, IEEE Transactions on, vol. AES-9, no. 4, pp. $499-503,1973$.

[17] H. Rasheed, F. Haroon, and N. Rajatheva, "Energy detection with diversity combining over $\mathrm{kg}$ fading for cognitive vanet," in 2016 IEEE 84th Vehicular Technology Conference (VTC-Fall), Sept 2016, pp. 1-5. 